\title{
Confocal laser endomicroscopy under propofol-based sedation for early gastric cancer and pre-cancerous lesions is associated with better diagnostic accuracy: a retrospective cohort study in China
}

\author{
Lihua Chu', Jialian Zhao², Cheng Sheng ${ }^{1,3}$, Min Yue ${ }^{4}$, Feifei Wang ${ }^{5}$, Shengwen Song ${ }^{1}$, Baoli Cheng ${ }^{1 * \dagger}$, \\ Guohao Xie ${ }^{1 * \dagger}$ and Xiangming Fang ${ }^{1}$
}

\begin{abstract}
Background: Confocal laser endomicroscopy (CLE) has advantages in detecting gastric neoplastic lesions, meanwhile it requires strict patient cooperation. Sedation could improve patient cooperation and quality of endoscopy. However, sedation is still not very popular in some resource-limited countries and regions. The purpose of this study was to compare propofol-based sedated versus un-sedated CLE in the value of diagnosing early gastric cancer (EGC) and precancerous lesions.

Methods: A retrospective, cohort, single center study of 226 patients who underwent CLE between January 1, 2015 and December 31, 2017 was performed. Patients enrolled were allocated into the propofol-based sedated group $(n=126)$ and the un-sedated group $(n=100)$. The comparison of validity and reliability of CLE for identifying $E G C$ and precancerous lesions between the two groups was performed through analyzing CLE diagnosis and pathological diagnosis. Reporting followed the STROBE guidelines.

Results: The area under receiver operating characteristic curve (AUROC) of diagnosing EGC in the sedated group was 0.97 (95\% Cl: 0.95 to 0.99), which was higher than that in the un-sedated group (0.88 ( $95 \%$ Cl: 0.80 to 0.97 ), $P=0.0407)$. CLE with sedation performed better than without sedation in diagnosing intraepithelial neoplasia and intestinal metaplasia ( $P=0.0008$ and $P=0.0001$, respectively). For patients considered as high-grade intraepithelial neoplasia or EGC by endoscopists, they would not get biopsy during CLE but receive endoscopic submucosal dissection (ESD) subsequently, and the misdiagnosis rate of CLE was $0 \%$ in the sedated group and $27.59 \%$ (95\% Cl: $10.30-44.91 \%)$ in the un-sedated group $(P=0.006)$.
\end{abstract}

Conclusions: Propofol based sedation was associated with improved diagnostic value of CLE for detecting EGC as well as precancerous lesions (intraepithelial neoplasia OR intestinal metaplasia).

Keywords: Propofol-based sedation, Confocal laser endomicroscopy, Early gastric cancer, Precancerous lesions

\footnotetext{
*Correspondence: chengbaoli1979@zju.edu.cn; 13958075805@126.com

${ }^{\dagger}$ Guohao Xie and Baoli Cheng have contributed equally to this study.

'Department of Anesthesiology, the First Affiliated Hospital, College of

Medicine, Zhejiang University, Zhejiang, Hangzhou, China

Full list of author information is available at the end of the article
}

(c) The Author(s). 2021 Open Access This article is licensed under a Creative Commons Attribution 4.0 International License, which permits use, sharing, adaptation, distribution and reproduction in any medium or format, as long as you give appropriate credit to the original author(s) and the source, provide a link to the Creative Commons licence, and indicate if changes were made. The images or other third party material in this article are included in the article's Creative Commons licence, unless indicated otherwise in a credit line to the material. If material is not included in the article's Creative Commons licence and your intended use is not permitted by statutory regulation or exceeds the permitted use, you will need to obtain permission directly from the copyright holder. To view a copy of this licence, visit http://creativecommons.org/licenses/by/4.0/ The Creative Commons Public Domain Dedication waiver (http://creativecommons.org/publicdomain/zero/1.0/] applies to the data made available in this article, unless otherwise stated in a credit line to the data. 


\section{Background}

Gastric cancer is one of the most common malignancy and leading causes of cancer mortality in the world [13], especially in East Asian countries [4, 5]. Detecting gastric malignancy at an early stage is vitally important, since early gastric cancer (EGC) may be curable, with reported 5-year survival rate of more than $90 \%$ [6]. Advanced gastric cancer usually has poor prognosis [6].

As the most useful tools for screening gastric cancer, a number of modern endoscopy devices and techniques, like confocal laser endomicroscopy (CLE), magnifying endoscopy and narrow-band image, have been developed to fulfill different diagnostic demands. Among these, CLE has advantages in detecting EGC and pre-malignant lesions, since it can provide a direct histological observation of the cells and subcellular regions in vivo, as well as demonstrate mucosal changes that cannot be detected by white light endoscopy (WLE) [7].

Sedation in gastroscopy is widely accepted in highincome countries, which are found to be associated with increased patient comfort and reduced complications related with poor patient cooperation, though it causes additional medical resource consumption [8]. As CLE procedures usually require prolonged endoscopy time and better patient cooperation, sedation is considered even indispensable in these patients $[9,10]$. However, whether sedation is associated with improved diagnostic quality of CLE is not well understood. Thus, evaluating the impact of sedation on CLE outcomes is still of value, especially in resource-limited countries and regions, such as China. Therefore, we conducted this retrospective, cohort study, to compare the diagnostic value for gastric superficial lesions, including EGC, intraepithelial neoplasia, and intestinal metaplasia between propofol based sedated and unsedated CLE in a university hospital within China.

\section{Methods \\ Study Design}

This was a single-center, retrospective, cohort study, analyzing outpatient probe-based CLE database and histopathology reports from the Department of Endoscopy Unit, the First Affiliated hospital, College of Medicine, Zhejiang University between January 1, 2015 and December 31, 2017. The research protocol was reviewed and accepted by the research ethics committee of the First Affiliated Hospital, College of Medicine, Zhejiang University on June 13, 2018 (Reference Number: 750/2017). The study followed the reporting guideline-Strengthening the Reporting of Observational Studies in Epidemiology (STROBE).

\section{Participants}

Patients enrolled were allocated into two groups according to their anesthesia type. Patients in the sedated group received a combination of propofol-based sedation and lidocaine-based pharyngeal anesthesia, while patients in the un-sedated group received lidocaine-based pharyngeal anesthesia only. Reports on juvenile patients ( $<18$ years old), or patients with advanced gastric cancer were excluded.

\section{Anesthetic procedure}

For all patients, after arrival, the electrocardiogram, noninvasive blood pressure and oxygen saturation were monitored. Lidocaine Hydrochloride mucilage (0.2 g/ 10ml; Jiangsu Jichuan Pharmaceutical Co., Jiangsu, China) was administered orally $5 \mathrm{~min}$ before the beginning of CLE for pharyngeal anesthesia. For patients with sedated CLE, a 20-gauge cannula was placed in a vein in the forearm, and sedation was induced with propofol (0.5 g/50ml; Xian Libang Pharmaceutical Co., Shanxi, China) $1.5-2 \mathrm{mg} / \mathrm{kg}$ intravenously. Sedation maintained was using 5-8 $\mathrm{mg} / \mathrm{kg} / \mathrm{h}$ propofol. Patients in the unsedated group received pharyngeal anesthesia only.

\section{CLE procedure}

CLE was conducted by two endoscopists who were with at least 5 years' experience in performing diagnostic CLE. The procedure involved the use of a cellvizio confocal miniprobe (CM-4880, GastroFlex ${ }^{\mathrm{Tm}} \mathrm{UHD}$, Mauna Kea, Paris, France), and a contrast agent (5ml of $10 \%$ fluorescein sodium; Alcon Laboratories,Inc. Fort Worth, USA).

CLE for all participants were performed according to the standard protocol. Each CLE procedure needed to be performed with no less than $20 \mathrm{~min}$. After a mucosal lesion was visualized by WLE, a total of $5 \mathrm{ml} 10 \%$ fluorescein sodium was administered intravenously. To obtain controlled CLE images, the probe was first gently contacted to normal mucosa around the lesion, ideally showing regular round or oval glands with homogeneous epithelial cells. The probe was subsequently moved to suspicious lesion to obtain CLE image, and following it, biopsies were obtained from the area. If the lesion was considered as high-grade intraepithelial neoplasia (HGIN) or EGC by the two endoscopists under CLE, the endoscopists would not get biopsy during the CLE procedure, but suggest patients for an endoscopic submucosal dissection (ESD) directly [6].

\section{CLE criteria and histopathological criteria}

The CLE criteria were based on the 2011 Miami classification [11]. Four CLE diagnoses were given through evaluating architecture of glands, cells and micro-vessels as follows: [1] normal mucosa or benign inflammatory lesions; [2] atrophy and/or intestinal metaplasia (IM); [3] intraepithelial neoplasia (IN), including low-grade intraepithelial neoplasia (LGIN) and HGIN; [4] cancer. 
The histopathological diagnostic criteria were based on the Updated Sydney System for classification and grading of gastritis [12] and the WHO classification of tumors [7]. EGC is defined as carcinoma confined to the mucosa or submucosa, regardless of lymph node metastatic status. Surgical or endoscopic ablation was recommended for neoplastic lesions. Endoscopic resection was selected for HGIN or mucosal carcinoma. Surgery was used for incomplete endoscopic resection [6].

\section{Data Collection}

Two trained research assistants collected the following data: patients' characteristics, CLE reports by endoscopists, pathological reports by pathologists. They also collected the information of patients who were strongly suspected for HGIN or EGC and further received ESD. These patients did not get biopsy during the CLE procedure, but had pathological results of ESD specimens. So the data of CLE reports and pathological diagnoses of ESD specimens were collected.

The complications appeared during CLE procedure were also recorded. Major complications were defined as need for intubation, intensive-care unit admission, resuscitation and death. Minor complications were defined as respiratory depression (Oxygen Saturation $\left(\mathrm{SaO}_{2}\right)<90 \%$ $>10 \mathrm{~s}$ ), hypotension (drop in systolic blood pressure of $>25 \%$ ), hypertension (raise in systolic blood pressure of $>25 \%$ ), bradycardia (drop in heart rate of $>20 \%$ ), or tachycardia ( $>100 \mathrm{bpm})$.

These data were entered into a Microsoft Excel 2013 (Microsoft Corporation, Washington, United States). Another two trained research assistants randomly extracted $10 \%$ of them to check the completeness, accuracy, and relevance of the information.

\section{Statistical analysis}

Enrolled patients were allocated into two groups: the sedated group and the un-sedated group. The diagnostic accuracy of intestinal metaplasia, intraepithelial neoplasia, gastric neoplasia lesions (IN OR EGC) and EGC that examined by CLE was compared between the two groups. Among them, IM and IN were defined as precancerous lesions.

No specific power calculation was performed, and the sample size of this study was determined by the number of patients recruited across site. Statistical analysis was performed by using the software STATA/MP 15.0 (Stata Inc., TX, USA). Numerical data were presented as numbers (percentage). The Pearson $x^{2}$ test was used to examine the significance of the association between two variables in a contingency table. Variables with a normal distribution were presented as mean \pm standard deviation (SD), and compared using analysis of $t$ test. A $P$ value of 0.05 (two-sided) was considered statistically significant.
The primary outcome was the comparison of the area under receiver operating characteristic curve (AUROC) of EGC and precancerous lesions that was diagnosed by CLE between the two groups. The gold standard for diagnosing EGC and precancerous lesions is the histopathological diagnosis. The nonparametric analysis was used for two-group comparison in AUROC.

The second outcome was the misdiagnosis rate of CLE. For patients without biopsy during CLE and received an ESD subsequently, the misdiagnosis rate of CLE was analyzed according to their pathological results of ESD specimens.

Another second outcome was the comparison of sensitivity, specificity, positive predictive value (PPV), negative predictive value (NPV) and kappa ( $\mathrm{K}$ ) value along with bionormal $95 \%$ confidence intervals $(95 \% \mathrm{CI})$ between the two groups. Agreement was regarded as poor withkvalue below 0.4 , good with value between 0.4 and 0.75 , and excellent withk value over 0.75 .

\section{Results}

Between January 1, 2015 and December 31, 2017, a total of 253 patients were eligible for study analysis, of which 27 (10 in the sedated group, 17 in the un-sedated group) was excluded because of the missing pathological data; the remaining 226 (126 in the sedated group, 100 in the un-sedated group) were analyzed. In this study, the most severe abnormality was used as each patient's diagnosis. For example, intestinal metaplasia associated with intraepithelial neoplasia was considered as intraepithelial neoplasia. When referring to histopathology, in the sedated group, 16 (12.70\%) patients were diagnosed as EGC, 27 $(21.43 \%)$ patients were diagnosed as intraepithelial neoplasia, 55 (43.65\%) patients were diagnosed as intestinal metaplasia and $28(22.22 \%)$ patients were diagnosed as normal mucosa or benign inflammatory lesions. In the un-sedated group, 18 (18.00\%) patients were diagnosed as EGC, 17 (17.00\%) patients were diagnosed as intraepithelial neoplasia, 36 (36.00\%) patients were diagnosed as intestinal metaplasia and $29(29.00 \%)$ patients were diagnosed as normal mucosa or benign inflammatory lesions. Detailed characteristics of the two groups are presented in Table 1.

The AUROC of CLE in diagnosing normal mucosa or benign inflammatory lesions in the sedated group was 0.80 (95\% CI: 0.71 to 0.89 ), which was higher than that in the un-sedated group (0.62 (95\% CI: 0.53 to 0.72$)$, $\mathrm{P}=0.0084$ ) (Fig. 1a).

When referred to precancerous lesions, the sedated group had higher AUROC of CLE in diagnosing intestinal metaplasia than the un-sedated group (0.83 (95\% CI: 0.76 to 0.89 ) VS 0.59 (95\% CI: 0.49 to 0.69 ), $P=$ 0.0001) (Fig. 1b). In diagnosing intraepithelial neoplasia, sedated CLE had higher AUROC (0.79 (95\% CI: 0.71 to 
Table 1 Patients' demographics

\begin{tabular}{lll}
\hline & $\begin{array}{l}\text { The sedated } \\
\text { group }\end{array}$ & $\begin{array}{l}\text { The un-sedated } \\
\text { group }\end{array}$ \\
\hline $\begin{array}{l}\text { Total number } \\
\text { Sex }\end{array}$ & 126 & 100 \\
Male & $77(61.11 \%)$ & $59(59.00 \%)$ \\
Female & $49(38.89 \%)$ & $41(41.00 \%)$ \\
Age & $58.10 \pm 10.69$ & $54.52 \pm 11.61$ \\
Histopathological diagnosis & & \\
Normal mucosa or benign & $28(22.22 \%)$ & $29(29.00 \%)$ \\
inflammatory lesions & & $36(36.00 \%)$ \\
IM & $55(43.65 \%)$ & $17(17.00 \%)$ \\
IN & $27(21.43 \%)$ & $18(18.00 \%)$ \\
EGC & $16(12.70 \%)$ & \\
\hline
\end{tabular}

IM Intestinal metaplasia; IN Intraepithelial neoplasia; EGC Early gastric cancer

0.87)) than un-sedated CLE (0.58 (95\% CI: 0.48 to 0.67) $(P=0.0008)$ (Fig. 1c).

While testing the value of CLE in diagnosing neoplastic lesions (EGC + intraepithelial neoplasia), the AUROC in the sedated group was 0.89 (0.83 to 0.95$)$, which was higher than that in the un-sedated group $(0.75(0.66$ to 0.84)) $(P=0.0073)$ (Fig. 1d). The AUROC of CLE in diagnosing EGC in the sedated group was 0.97 (95\% CI: 0.95 to 0.99 ), and in the un-sedated group was 0.88 (95\% CI: 0.80 to 0.97$)(P=0.0407)$ (Fig. 1e).The sensitivity, specificity, PPV and NPV and $\mathrm{K}$ of intestinal metaplasia, intraepithelial neoplasia, neoplastic lesions
(EGC + intraepithelial neoplasia) and EGC were better in sedated CLE group than in un-sedated CLE group. Detailed information was listed in Table 2.

To those who received ESD, the agreement between main CLE finding and the ESD pathological diagnose was assessed. A total of 24 patients in the sedated group and 29 in the un-sedated group were suggested for ESD directly. The misdiagnosis rate of CLE in the sedated group was $0 \%$, and in the un-sedated group was $27.59 \%$ (95\% CI: $10.30-44.91 \%)$, which had significant difference $(P=0.006)$.

Minor complication of hypotension was found in two patients with sedated CLE. Three patients and one patient in the un-sedated group suffered tachycardia and hypertension, respectively. There were no major complications found in the two groups.

\section{Discussion}

Our results showed that CLE with propofol-based sedation had remarkably better discrimination for diagnosing EGC and pre-malignant lesions (intestinal metaplasia and intraepithelial neoplasia) than that of un-sedated CLE. And for patients without biopsy during CLE procedure and received an ESD directly, the misdiagnosis rate of CLE was significantly lower in the sedated group than in the un-sedated group according to the final pathological results. To the best of our knowledge, this is the first study to demonstrate such a positive association between sedation and CLE outcomes.
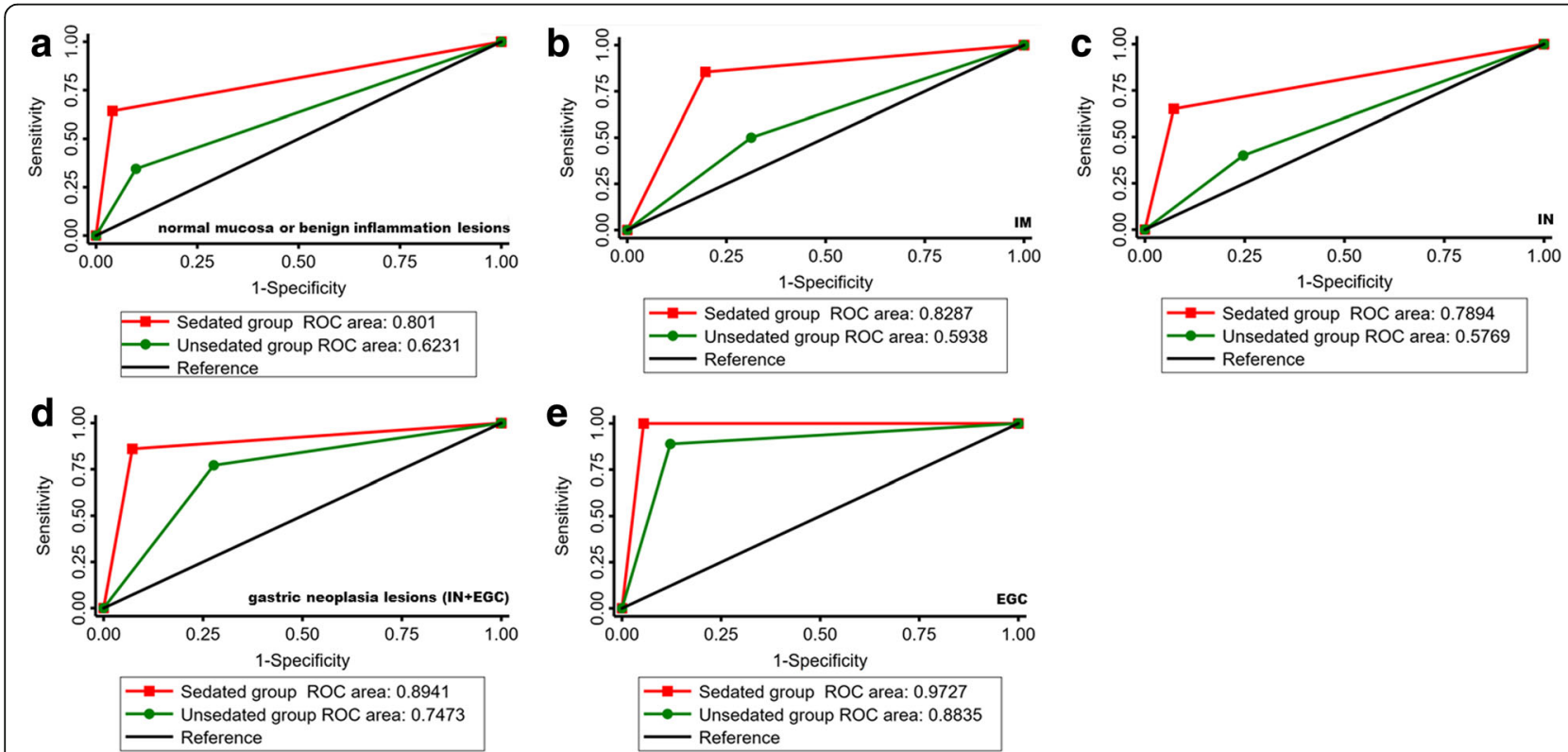

Fig. 1 Comparison of AUROC of CLE for diagnosing different types of gastric lesions between the two groups. The value of CLE was better in the sedated CLE group than in the un-sedated CLE group in diagnosing normal mucosa or benign inflammation lesions $(P=0.0084)(\mathbf{a})$, intestinal metaplasia $(P=0.0001)(\mathbf{b})$, intraepithelial neoplasia $(P=0.0008)(\mathbf{c})$, gastric neoplasia lesions $(\mathrm{IN}+\mathrm{EGC})(P=0.0073)(\mathbf{d})$ and EGC $(P=0.0407)$ (e). AUROC, The area under receiver operating characteristic curve; CLE, Confocal laser endomicroscopy; EGC, Early gastric cancer 
Table 2 Assessment of diagnostic value for gastric mucosal lesions based on CLE in the two groups

\begin{tabular}{|c|c|c|c|c|c|}
\hline & Sensitivity $(\%,(\mathrm{Cl}))$ & Specificity $(\%,(\mathrm{Cl}))$ & PPV $(\%,(\mathrm{Cl}))$ & NPV $(\%,(C I))$ & kvalue \\
\hline \multicolumn{6}{|c|}{ Normal mucosa or benign inflammatory lesions } \\
\hline The sedated group & $64.29(44.11$ to 80.69$)$ & $95.92(89.28$ to 98.68$)$ & $81.82(58.99$ to 94.01$)$ & $90.38(82.62$ to 95.04$)$ & 0.65 \\
\hline The un-sedated group & $\begin{array}{l}34.48(18.60 \text { to } 54.34) \\
\text { (18.60 to } 54.33)\end{array}$ & $90.14(80.16$ to 95.61$)$ & $58.82(33.45$ to 80.57$)$ & $77.11(66.34$ to 85.32$)$ & 0.28 \\
\hline \multicolumn{6}{|l|}{ IM } \\
\hline The sedated group & $85.55(72.78$ to 93.07$)$ & $80.28(68.80$ to 88.43$)$ & $77.05(64.20$ to 86.46$)$ & $87.77(76.64$ to 94.16$)$ & 0.65 \\
\hline The un-sedated group & $50.00(33.22$ to 66.78$)$ & $68.75(55.80$ to 79.43$)$ & $47.37(31.31$ to 63.95$)$ & 70.97(57.87 to 81.45$)$ & 0.19 \\
\hline \multicolumn{6}{|l|}{ IN } \\
\hline The sedated group & $65.11(49.01$ to 78.55$)$ & $92.78(84.35$ to 97.03$)$ & $82.35(64.83$ to 92.61$)$ & $83.70(74.21$ to 90.29$)$ & 0.61 \\
\hline The un-sedated group & $40.00(24.35$ to 57.79$)$ & $75.39(62.87$ to 84.87$)$ & $46.67(28.80$ to 65.36$)$ & $70.00(57.71$ to 80.07$)$ & 0.16 \\
\hline \multicolumn{6}{|l|}{ IN + EGC } \\
\hline The sedated group & $86.05(71.37$ to 94.20$)$ & $92.77(84.35$ to 97.03$)$ & $86.05(71.37$ to 94.20$)$ & $92.77(84.35$ to 97.03$)$ & 0.79 \\
\hline The un-sedated group & $77.14(59.45$ to 88.96$)$ & $72.31(59.61$ to 82.35$)$ & $60.00(44.37$ to 73.94$)$ & $85.46(72.78$ to 93.07$)$ & 0.46 \\
\hline \multicolumn{6}{|l|}{ EGC } \\
\hline The sedated group & $100(75.93$ to 100$)$ & $94.55(88.02$ to 97.76$)$ & $72.73(49.56$ to 88.39$)$ & $100(95.56$ to 100$)$ & 0.82 \\
\hline The un-sedated group & $88.89(63.93$ to 68.05$)$ & $87.80(78.27$ to 93.68$)$ & $61.54(40.73$ to 79.09$)$ & $97.30(89.69$ to 99.53$)$ & 0.65 \\
\hline
\end{tabular}

Sedation is a drug-induced depression in the level of consciousness, which is recommended for GI endoscopy [13]. It could relieve the patients' anxiety and discomfort, diminish the patients' memory of the event and improve the outcome of examination [14]. Our study showed sedated CLE had a better diagnostic value for EGC than un-sedated CLE. The results of the sedated group were similar with the previous study, whose sensitivity and specificity of diagnosing EGC was 88.1 and $98.6 \%$ respectively [6], but results in the un-sedated group seems not so satisfactory. This may due to three reasons: Firstly, patients with sedation are able to well tolerate the CLE procedure [13] and endure inflation of the stomach to a greater extent, compared to patients without sedation [15]; Secondly, adequate level of sedation in CLE may improve the efficiency and quality of the procedure by providing the endoscopist with optimal conditions for a thorough visualization, while eliminating any distraction due to an uncomfortable patient [16]; Thirdly, with sedation, the examining time can be prolonged without patient complaints if needed [15]. The operator of CLE would be more focused and confident during the examinations and in no hurry to finish the procedure.

Gastric cancer is believed to arise from a series of premalignant lesions, through a number of stages from chronic atrophic gastritis, by way of intestinal metaplasia, through LGIN and HGIN, up to cancer [17]. A large sample study showed that approximately 1 in 39 with intestinal metaplasia and 1 in 19 with dysplasia would progress to gastric cancer within 20 years [18]. Considering the higher incidence of intestinal metaplasia and intraepithelial neoplasia compared with EGC, especially in high-risk regions like China, it is desirable to explore whether CLE with sedation could improve the diagnostic value of identifying precancerous lesions [19]. The ROC curve analysis revealed that sedation could increase the AUROC of CLE diagnosing intestinal metaplasia and intraepithelial neoplasia. It is important because immediate diagnosis as well as precise biopsy can help endoscopist to make a quick decision for treatment, especially for intraepithelial neoplasia at a high grade.

In the current study, propofol based sedation was adopted, which makes endoscopy almost painless, with a very predictable, rapid recovery process and improved patient satisfaction [20, 21]. Compared with benzodiazepines and opioids, sedation with propofol can improve the quality of endoscopy, such as increasing the detecting rate of advanced lesions [22] and polyp [16]. Sedation is considered with an added risk of complications, but the complication and mortality rates of a prospective research, involving 191,242 endoscopies with propofol sedation, were 0.04 and $0.003 \%$, respectively [24]. In addition, a recent large multicenter registry study, with 300000 patients enrolled, confirmed that severe acute sedation-related complications are rare during GI endoscopy with a very low mortality [25].

Although sedated endoscopy is widely accepted in high-income countries and regions [14], it is not so popular in resource-limited countries and regions [26]. For example, in the studied tertiary hospital, which is located in a wealthier area of China, the ratio of sedation in gastroscopy was only $43.2 \%$ in 2018. The limited staffing of anesthesiologists is the main restriction factor. 
Although extensive data have demonstrated the safety and efficacy of non-anesthesiologist-administered propofol sedation, the American Society of Anesthesiologists continues to maintain that propofol sedation should be performed only by anesthesia providers [23, 27]. The economic factor also hinders the development of sedated CLE in China. Our study showed sedation could improve the diagnostic ability of EGC and pre-malignant lesions. Thus, it might be better for government to put more medical resources and provide more medical insurance supports in the field of sedated CLE. And the publicity of sedation should be further strengthened by the hospital administration as well as relevant medical departments.

Our study was limited by a few factors. Firstly, its retrospective study design was the main weakness. A prospective validation study is needed to confirm the association between sedation and improved quality of CLE. Furthermore, in the validation phase, we can also assess the safety and patients satisfactory of sedation. Secondly, the procedure time was not recorded in our databases. The procedure of CLE with propofol-based sedation might be prolonged for the patient's great cooperation. The longer time might be correlated with the quality of mucosal inspection during CLE.

\section{Conclusions}

In summary, this is the first study to validate the propofol-based sedation in improving the value of CLE in diagnosing EGC and precancerous lesions. Our results showed the improvement of validity and reliability of CLE in diagnosing gastric superficial cancerous and precancerous lesions though sedation. It indicates that, especially in resource-limited countries and regions, more patient undergoing CLE would benefit from sedation if more medical resources were put into this area.

\section{Abbreviations \\ 95\% Cl: $95 \%$ confidence intervals; AUROC: Area under receiver operating characteristic curve; CLE: Confocal laser endomicroscopy; EGC: Early gastric cancer; ESD: Endoscopic submucosal dissection; HGIN: High-grade intraepithelial neoplasia; IM: Intestinal metaplasia; IN: Intraepithelial neoplasia; LGIN: Low-grade intraepithelial neoplasia; NPV: Negative predictive value; PPV: Positive predictive value; ROC: Receiver operating characteristic; SD: Standard deviation; WLE: White light endoscopy}

\section{Acknowledgements}

None.

\section{Authors' contributions}

LHC wrote the manuscript. JLZ did the analysis of the data. CS and MY collected the data. FFW and SWS extracted $10 \%$ of the data to check the completeness, accuracy, and relevance of the information. XMF made the critical revision of the article for important intellectual content. GHX and BLC gave the conception and design, and made the final approval of article. All authors reviewed and approved the final version of the manuscript.

\section{Funding}

None.

\section{Availability of data and materials}

The datasets generated and analyzed during the present study are available from the corresponding author on reasonable request.

\section{Declarations}

Ethics approval consent to participate

This study was approved by the research ethics committee of the First Affiliated Hospital, College of Medicine, Zhejiang University on June 13, 2018 (Reference Number: 750/2017). Because of retrospective study, informed consent was waived.

\section{Consent for publication}

Not applicable.

\section{Competing interests}

The authors declare that there are no conflicts of interest in this work.

\section{Author details}

'Department of Anesthesiology, the First Affiliated Hospital, College of Medicine, Zhejiang University, Zhejiang, Hangzhou, China. ${ }^{2}$ Department of Anesthesiology, the Children's Hospital, College of Medicine, Zhejiang University, Hangzhou, China. ${ }^{3}$ Department of Anesthesiology, the First Affiliated Hospital, College of Medicine, Zhejiang University, Beilun District, Ningbo, China. ${ }^{4}$ Department of Gastroenterology, the First Affiliated Hospital, College of Medicine, Zhejiang University, Hangzhou, China. ${ }^{5}$ Department of Intensive Care Unit, the First Affiliated Hospital, College of Medicine, Zhejiang University, Hangzhou, China.

Received: 8 July 2020 Accepted: 21 March 2021

Published online: 30 March 2021

\section{References}

1. Bray F, Ferlay J, Soerjomataram I, Siegel RL, Torre LA, Jemal A. Global cancer statistics 2018: GLOBOCAN estimates of incidence and mortality worldwide for 36 cancers in 185 countries. Cancer J Clin. 2018;68(6):394-424.

2. Zhang HP, Yang S, Chen WH, Hu TT, Lin J. The diagnostic value of confocal laser endomicroscopy for gastric cancer and precancerous lesions among Asian population: a system review and meta-analysis. Scand J Gastroenterol. 2017:52(4):382-8.

3. Murray CJ, Vos T, Lozano R, Naghavi M, Flaxman AD, Michaud C, et al. Disability-adjusted life years (DALYS) for 291 diseases and injuries in 21 regions, 1990-2010: a systematic analysis for the Global Burden of Disease Study 2010. Lancet. 2012;380(9859):2197-223.

4. Chen W, Zheng R, Zhang S, Zhao P, Zeng H, Zou X. Report of cancer incidence and mortality in China, 2010. Annals of translational medicine. 2014;2(7):61

5. Pourhoseingholi MA, Vahedi M, Baghestani AR. Burden of gastrointestinal cancer in Asia; an overview. Gastroenterology and hepatology from bed to bench. 2015;8(1):19-27.

6. Li WB, Zuo XL, Li CQ, Zuo F, Gu XM, Yu T, et al. Diagnostic value of confocal laser endomicroscopy for gastric superficial cancerous lesions. Gut. 2011 60(3):299-306.

7. Stolte M. The new Vienna classification of epithelial neoplasia of the gastrointestinal tract: advantages and disadvantages. Virchows Archiv: an international journal of pathology. 2003;442(2):99-106.

8. $S$ G, ZZ G. Advance in clinical application of probe-based confocal laser endomicroscopy. Chin J Gastroenterol. 2014;19:43-6.

9. Kang D, Do D, Ryu J, Grant CN, Giddings SL, Rosenberg M, et al. A miniaturized, tethered, spectrally-encoded confocal endomicroscopy capsule. Lasers in surgery and medicine. 2019.

10. Zuo XL, Li Z, Li CQ, Zheng YY, Xu LD, Chen J, et al. Probe-based endomicroscopy for in vivo detection of gastric intestinal metaplasia and neoplasia: a multicenter randomized controlled trial. Endoscopy. 2017; 49(11):1033-42.

11. Wallace M, Lauwers GY, Chen Y, Dekker E, Fockens P, Sharma P, et al. Miami classification for probe-based confocal laser endomicroscopy. Endoscopy. 2011;43(10):882-91.

12. Dixon MF, Genta RM, Yardley JH, Correa P. Classification and grading of gastritis. The updated Sydney System. International Workshop on the 
Histopathology of Gastritis, Houston 1994. The American journal of surgical pathology. 1996;20(10):1161-81.

13. Committee ASoP, Early DS, Lightdale JR, Vargo JJ 2nd, Acosta RD, Chandrasekhara V, et al. Guidelines for sedation and anesthesia in Gl endoscopy. Gastrointest Endosc. 2018;87(2):327-37.

14. Vaessen HH, Knape JT. Considerable Variability of Procedural Sedation and Analgesia Practices for Gastrointestinal Endoscopic Procedures in Europe. Clinical endoscopy. 2016;49(1):47-55.

15. Meining A, Semmler V, Kassem AM, Sander R, Frankenberger U, Burzin M, et al. The effect of sedation on the quality of upper gastrointestinal endoscopy: an investigator-blinded, randomized study comparing propofol with midazolam. Endoscopy. 2007;39(4):345-9.

16. Abu Baker F, Mari A, Aamarney K, Hakeem AR, Ovadia B, Kopelman Y. Propofol sedation in colonoscopy: from satisfied patients to improved quality indicators. Clinical experimental gastroenterology. 2019:12:105-10.

17. Januszewicz W, Wieszczy P, Bialek A, Karpinska K, Szlak J, Szymonik J, et al. Endoscopist biopsy rate as a quality indicator for outpatient gastroscopy: a multicenter cohort study with validation. Gastrointest Endosc. 2019;89(6):1141-9.

18. Song $H$, Ekheden IG, Zheng Z, Ericsson J, Nyren O, Ye W. Incidence of gastric cancer among patients with gastric precancerous lesions: observational cohort study in a low risk Western population. Bmj. 2015; 351:h3867.

19. Li Z, Yu T, Zuo XL, Gu XM, Zhou CJ, Ji R, et al. Confocal laser endomicroscopy for in vivo diagnosis of gastric intraepithelial neoplasia: a feasibility study. Gastrointest Endosc. 2010;72(6):1146-53.

20. Nishizawa T, Suzuki H. Propofol for gastrointestinal endoscopy. United European gastroenterology journal. 2018;6(6):801-5.

21. Singh H, Poluha W, Cheung M, Choptain N, Baron KI, Taback SP. Propofol for sedation during colonoscopy. The Cochrane database of systematic reviews. 2008(4):CD006268

22. Wang A, Hoda KM, Holub JL, Eisen GM. Does level of sedation impact detection of advanced neoplasia? Digestive diseases sciences. 2010;55(8): 2337-43.

23. Sidhu R, Turnbull D, Newton M, Thomas-Gibson S, Sanders DS, Hebbar S, et al. Deep sedation and anaesthesia in complex gastrointestinal endoscopy: a joint position statement endorsed by the British Society of Gastroenterology (BSG), Joint Advisory Group (JAG) and Royal College of Anaesthetists (RCOA). Frontline gastroenterology. 2019:10(2):141-7.

24. Sharma VK, Nguyen CC, Crowell MD, Lieberman DA, de Garmo P, Fleischer DE. A national study of cardiopulmonary unplanned events after $\mathrm{Gl}$ endoscopy. Gastrointest Endosc. 2007;66(1):27-34.

25. Behrens A, Kreuzmayr A, Manner H, Koop H, Lorenz A, Schaefer C, et al. Acute sedation-associated complications in Gl endoscopy (ProSed 2 Study): results from the prospective multicentre electronic registry of sedationassociated complications. Gut. 2018.

26. Wang HL, Ye F, Liao WF, Xia B, Zheng GR. Unsedated versus sedated gastrointestinal endoscopy: a questionnaire investigation in Wuhan, central China. Journal of Huazhong University of Science and Technology Medical sciences $=$ Hua zhong ke ji da xue xue bao Yi xue Ying De wen ban = Huazhong keji daxue xuebao Yixue Yingdewen ban. 2013;33(6):857-61.

27. Perel A. Non-anaesthesiologists should not be allowed to administer propofol for procedural sedation: a Consensus Statement of 21 European National Societies of Anaesthesia. Eur J Anaesthesiol. 2011;28(8):580-4.

\section{Publisher's Note}

Springer Nature remains neutral with regard to jurisdictional claims in published maps and institutional affiliations.

Ready to submit your research? Choose BMC and benefit from:

- fast, convenient online submission

- thorough peer review by experienced researchers in your field

- rapid publication on acceptance

- support for research data, including large and complex data types

- gold Open Access which fosters wider collaboration and increased citations

- maximum visibility for your research: over $100 \mathrm{M}$ website views per year

At $\mathrm{BMC}$, research is always in progress.

Learn more biomedcentral.com/submissions 